\title{
ASYMMETRIC BIFURCATION
}

\section{S. ROSENBLAT}

(Received 31 October 1978)

(Revised 22 January 1979)

\begin{abstract}
A study is made of a non-linear diffusion equation which admits bifurcating solutions in the case where the bifurcation is asymmetric. An analysis of the initial-value problem is made using the method of multiple scales, and the bifurcation and stability characteristics are determined. It is shown that a suitable interpretation of the results can lead to determination of the choice of the bifurcating solution adopted by the system.
\end{abstract}

\section{Introduction}

In a recent paper, Benjamin [1] has argued strongly that symmetric bifurcations having the form shown in Fig. 1(a) rarely occur in nature. An inhomogeneity in a physical system may cause a departure from symmetry and the resulting bifurcation picture may well be more likely to have the structure shown in Fig. 1(b). It is certainly true that there are many known instances when Fig. 1(b) applies rather than Fig. 1(a). The classical Bénard convection problem in fluid mechanics, for example, results in symmetric supercritical bifurcation, but factors such as variations in fluid properties and surface tension gradients transform the situation into the asymmetric picture of Fig. 1(b).

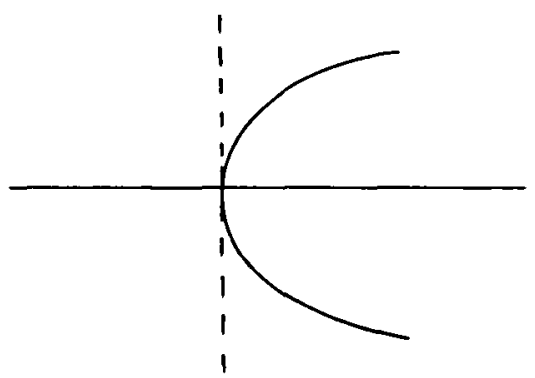

(a) Symmetric bifurcation

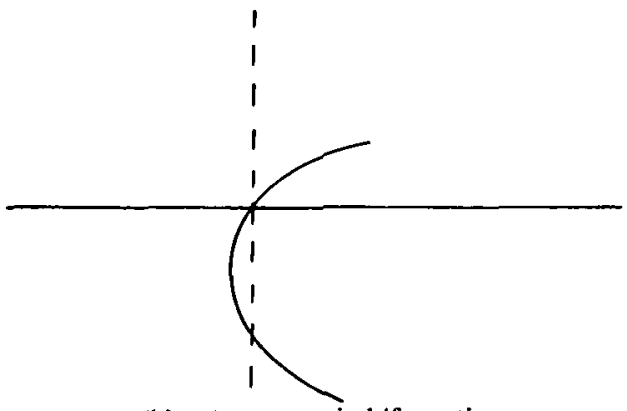

(b) Asymmetric bifurcation

Fig. 1 
Our object in this paper is to outline a simple technique for dealing with asymmetric bifurcations. By way of motivation, consider the boundary-value problem

$$
\left.\begin{array}{rl}
v^{\prime \prime}+\lambda v-a v^{2}-v^{3} & =0, \quad 0<x<\pi, \\
v(0) & =v(\pi)=0 .
\end{array}\right\}
$$

where $\lambda, a$ are real constants, and where a prime denotes differentiation with respect to $x$. The eigenvalues of the linearized problem occur at the points $\lambda=n^{2}, n=1,2,3, \ldots$, and the structure of the equation suggests that the bifurcation curve emanating from each eigenvalue may have the form shown in Fig. 1(b).

Confining our attention to the neighbourhood of the first eigenvalue $\lambda=1$, we can determine the bifurcating solution by applying the standard PoincaréLinstedt method. We introduce a real parameter $\varepsilon$ whose norm is a measure of the norm of the bifurcating solution, and write

$$
\left.\begin{array}{rl}
v(x) & =\varepsilon v_{1}(x)+\varepsilon^{2} v_{2}(x)+\ldots, \\
\lambda-1 & =\varepsilon \lambda_{1}+\varepsilon^{2} \lambda_{2}+\ldots .
\end{array}\right\}
$$

Substituting (1.2) into (1.1) and equating to zero coefficients of like powers of $\varepsilon$, we obtain a sequence of boundary-value problems as follows:

$$
\begin{array}{ll}
v_{1}^{\prime \prime}+v_{1}=0, & v_{1}(0)=v_{1}(\pi)=0, \\
v_{2}^{\prime \prime}+v_{2}=-\lambda_{1} v_{1}+a v_{1}^{2}, & v_{2}(0)=v_{2}(\pi)=0, \\
v_{3}^{\prime \prime}+v_{3}=-\lambda_{2} v_{1}-\lambda_{1} v_{2}+2 a v_{1} v_{2}+v_{1}^{3}, & v_{3}(0)=v_{3}(\pi)=0,
\end{array}
$$

and similarly for higher order terms. Equation (1.3) has the solution

$$
v_{1}(x)=\sin x
$$

Application of the Fredholm alternative now shows that (1.4) has a solution if its right-hand side is orthogonal to $\sin x$. This requirement gives

$$
\lambda_{1}=\frac{8 a}{3 \pi}
$$

and the solution of (1.4) is

$$
v_{2}(x)=\frac{a}{6}\left(3+\cos 2 x+\frac{8}{\pi} x \cos x-4 \cos x\right) .
$$

The Fredholm alternative applied to the right-hand side of (1.5) yields the expression

$$
\lambda_{2}=\frac{3}{4}+\gamma a^{2},
$$

where $\gamma$ is a constant obtainable from elementary integrations. 
To order $\varepsilon^{2}$ the bifurcation curve is now seen to have the form

$$
i-1=\frac{8 a}{3 \pi} \varepsilon+\left(\frac{3}{4}+\gamma a^{2}\right) \varepsilon^{2} .
$$

We observe that the second term on the right-hand side of (1.10) is much smaller than the first unless $a$ is itself very small, specifically, unless $a$ and $\varepsilon$ are comparable. If $a$ is large compared with $\varepsilon,(1.10)$ describes a curve in the $(\lambda, \varepsilon)$ plane which is a slight departure from a straight line through the bifurcation point $\lambda=1, \varepsilon=0$. In this case (1.10) does not extend to the turn-around point of the bifurcation curve in Fig. 1(b), and therefore does not describe the most interesting feature of the problem (1.1). If, on the other hand, $a=O(\varepsilon)$, then (1.10) does describe the turn-around of the curve.

Since the quantity $a$ is a measure of the degree of asymmetry, it follows from the preceding discussion that a perturbation technique gives the most useful results when the asymmetry is small. There are, in fact, many practical situations in which asymmetry is small; the convection problems mentioned earlier belong to this category. But when this is the case it should be possible to utilize the smallness of the asymmetry from the outset, and we propose to show in this paper how this can be done. A parameter is introduced to measure the asymmetry, and a perturbation based on the smallness of this parameter is developed. In this work we demonstrate the technique by treating a particular model nonlinear diffusion equation; the extension to more general problems, however, is fairly evident. In addition to computing the solution branches we shall use the same perturbation to determine their stability and to discuss their evolution from given initial states.

We shall consider the following non-linear partial differential equation:

$$
\frac{\partial v}{\partial t}=\frac{\partial^{2} v}{\partial x^{2}}+\lambda v-\varepsilon a v^{2}-v^{3}
$$

in the domain $t>0,0<x<\pi$. Here $\lambda, \varepsilon$ and $a$ are real parameters where, without loss of generality, we take $\varepsilon>0$ and $a>0$. We shall look for solutions in the form of perturbation expansions for $\varepsilon$ sufficiently small. Equation (1.11) is subject to the boundary conditions

$$
v(0, t)=v(\pi, t)=0, \quad t>0,
$$

together with initial conditions which will be specified later.

In Section 2 we solve the initial-value problem using a multiple scaling technique. The solution obtained in this way enables us to determine the bifurcating branches and their stability. In Section 3, we discuss the important role that asymmetry plays in determining which of two stable branches a dynamical system chooses. We shall show that, in a situation like Fig. 1(b), the lower branch is in a specific sense "more stable" than the upper branch, and is therefore the one more likely to be selected by the system. 


\section{Evolution of solutions}

We outline now a method of solving the boundary-value problem (1.11)-(1.12) together with the initial condition

$$
v(x, 0)=\varepsilon h(x)=\varepsilon \sum_{k=1}^{\infty} h_{k} \sin k x .
$$

We follow closely the procedure used by Matkowsky [3] and introduce a second, slow time scale defined by

$$
\tau=\varepsilon^{2} t
$$

Equation (1.11) then takes the form

$$
\frac{\partial v}{\partial t}+\varepsilon^{2} \frac{\partial v}{\partial \tau}=\frac{\partial^{2} v}{\partial x^{2}}+\lambda v-\varepsilon a v^{2}-v^{3}
$$

and we assume the solution to have the structure

$$
v(x, t, \tau, \varepsilon)=\varepsilon\left[u_{0}(x, t, \tau)+\varepsilon^{2} u_{1}(x, t, \tau)+\ldots\right] .
$$

Set $\lambda=1+\varepsilon^{2} \mu$, substitute (2.4) into (2.3) and equate coefficients of like powers of $\varepsilon$. We obtain a sequence of equations, the first two of which are

$$
-\frac{\partial u_{0}}{\partial t}+\frac{\partial^{2} u_{0}}{\partial x^{2}}+u_{0}=0
$$

and

$$
-\frac{\partial u_{1}}{\partial t}+\frac{\partial^{2} u_{1}}{\partial x^{2}}+u_{1}=\frac{\partial u_{0}}{\partial \tau}-\mu u_{0}+a u_{0}^{2}+u_{0}^{3} .
$$

The boundary and initial conditions are respectively

and

$$
u_{0}=0, \quad u_{1}=0 \quad \text { on } x=0, \pi,
$$

$$
u_{0}(x, 0,0)=h(x), \quad u_{1}(x, 0,0)=0 .
$$

The solution of (2.5) satisfying the boundary conditions is

$$
u_{0}(x, t, \tau)=A_{01}(\tau) \sin x+\sum_{k=2}^{\infty} A_{0 k}(\tau) \exp \left(-\sigma_{k} t\right) \sin k x,
$$

where $\sigma_{k}=k^{2}-1>0$ for $k=2,3, \ldots$. The slowly varying functions $A_{0 k}(\tau)$ must satisfy the initial conditions

$$
A_{\mathrm{ok}}(0)=h_{k}, \quad k=1,2, \ldots
$$

The next step in the procedure is to substitute for $u_{0}$ from (2.9) in the right-hand side of (2.6), and to determine the functions $A_{0 k}(\tau)$ such that the solution $u_{1}$ 
satisfies the boundary conditions and is bounded on the fast time scale. Since the terms corresponding to $k \geqslant 2$ in (2.9) are damped on the fast time scale, they have no effect on the boundedness of $u_{1}$. Thus we can write (2.6) in the form

$$
\begin{array}{r}
-\frac{\partial u_{1}}{\partial t}+\frac{\partial^{2} u_{1}}{\partial x^{2}}+u_{1}=\frac{d A_{01}}{d \tau} \sin x-\mu A_{01} \sin x+a A_{01}^{2} \sin ^{2} x+A_{01}^{3} \sin ^{3} x \\
\text { +exponentially damped terms. }
\end{array}
$$

It is clear now that the equation determining $A_{01}$ is obtained by applying the Fredholm alternative to the explicitly stated terms on the right-hand side of (2.11). (See [3] for further discussion of this point.) In this manner we obtain for $A_{01}(\tau)$ the initial-value problem:

$$
\frac{d A_{01}}{d \tau}-\mu A_{01}+\frac{8 a}{3 \pi} A_{01}^{2}+\frac{3}{4} A_{01}^{3}=0, \quad A_{01}(0)=h_{1} .
$$

Equation (2.12) is a generalization of the celebrated Landau equation which has been used to describe the evolution of disturbances in fluid dynamics stability problems. An equation of the form (2.12) has been proposed in the study of viscosity variations [4] and surface curvature [2] in the Bénard convection problem.

Equation (2.12) can be written in the form

$$
\frac{d A_{01}}{d \tau}+\frac{3}{4} A_{01}\left(A_{01}-A_{0}^{(1)}\right)\left(A_{01}-A_{0}^{(2)}\right)=0, \quad A_{01}(0)=h_{1},
$$

where $A_{0}^{(1)}, A_{0}^{(2)}$ are given by

$$
A_{0}^{(1)}=-\frac{16 a}{9 \pi}+\sqrt{\left[\left(\frac{16 a}{9 \pi}\right)^{2}+\frac{4}{3} \mu\right]}, \quad A_{0}^{(2)}=-\frac{16 a}{9 \pi}-\sqrt{\left[\left(\frac{16 a}{9 \pi}\right)^{2}+\frac{4}{3} \mu\right]} .
$$

Clearly the solutions $A_{0}^{(1)}$ and $A_{0}^{(2)}$ exist only when

$$
\mu>-\frac{3}{4}\left(\frac{16 a}{9 \pi}\right)^{2}
$$

they coalesce when (2.15) becomes an equality.

The steady-state solutions of (2.13) are obviously the null solution, and the solutions $A_{0}^{(1)}, A_{0}^{(2)}$ when (2.15) holds. Although the general solution to the initialvalue problem (2.13) can be written down, it is unnecessary to do so, since its behaviour can be inferred from the following considerations. When $\mu>0$ we have that $A_{0}^{(1)}>0>A_{0}^{(2)}$. If $h_{1}>A_{0}^{(1)}$, the gradient $A_{01}^{\prime}$ is initially negative, and remains negative for all $\tau \geqslant 0$. The solution therefore approaches $A_{0}^{(1)}$ asymptotically as $\tau \rightarrow \infty$. If $0<h_{1}<A_{0}^{(1)}$, the gradient is initially positive and remains positive, and the solution again tends to $A_{0}^{(1)}$ as $\tau \rightarrow \infty$. Thus for $\mu>0$ and $h_{1}>0$ the solution approaches $A_{0}^{(1)}$ asymptotically as $\tau \rightarrow \infty$; the approach 
can be shown to be exponential. By similar reasoning we can see that for $\mu>0$ and $h_{1}<0$ the solution tends to $A_{0}^{(2)}$ as $\tau \rightarrow \infty$. Hence we see that $A_{0}^{(1)}, A_{0}^{(2)}$ are both stable solutions when $\mu>0$.

In the subcritical regime, $-\frac{3}{4}(16 a / 9 \pi)^{2}<\mu<0$, the situation is a little different. Here we have that $0>A_{0}^{(1)}>A_{0}^{(2)}$. Hence if $h_{1}>0$ the gradient is negative for all $\tau \geqslant 0$ and the solution tends to the null solution. If $0>h_{1}>A_{0}^{(1)}$, the gradient is positive and the solution increases towards 0 as $\tau \rightarrow \infty$. When $A_{0}^{(1)}>h_{1}>A_{0}^{(2)}$ the gradient is negative and the solution decreases towards $A_{0}^{(2)}$. Finally, when $A_{0}^{(2)}>h_{1}$ the gradient is positive and the solution approaches $A_{0}^{(2)}$ asymptotically as $\tau \rightarrow \infty$. Thus, in the subcritical regime 0 and $A_{0}^{(2)}$ are stable solutions.

Although we have not attempted to do so here, it is possible to show rigorously that the results stated in the two preceding pargraphs are valid in the sense of first approximations for small $\varepsilon$.

\section{Discussion}

We have seen in the preceding sections that when $\mu>\hat{\mu}$, where

$$
\hat{\mu}=-\frac{3}{4}\left(\frac{16 a}{9 \pi}\right)^{2}
$$

the system has three solutions (except when $\mu=0$ ); one of these solutions is unstable while the other two are asymptotically stable in the linear approximation. The question then arises naturally, as $\mu$ increases towards and beyond the bifurcation point, does the system prefer one or other of the two stable solutions or are they equally likely to occur? Linear stability theory, which is concerned with infinitesimal disturbances, cannot answer this question since it provides no information regarding domains of attraction of stable solutions. Recently Weinberger and Rosenblat [5] discussed this issue with respect to a dynamical system subject to random noise, and indicated a possible answer in terms of the probability that the system would eventually choose one of the stable solutions. The analysis of Section 2, however, enables us to discuss the question in deterministic terms.

When $\mu<\hat{\mu}$ the system "ocupies" the null solution which is in fact globally stable. Suppose that $\mu$ is increased very gradually, and consider the behaviour of the system when $\hat{\mu}<\mu<0$. For each $\mu \in(\hat{\mu}, 0)$ there are disturbances which will displace the system from the null solution and cause it to move towards the lower branch $A_{0}^{(2)}$. Specifically, these are disturbances for which $h_{1}$ is negative with $\left|h_{1}\right|>\left|A_{0}^{(1)}\right|$. Since $A_{0}^{(1)} \rightarrow 0$ as $\mu \rightarrow \infty$, the magnitude of $h_{1}$ at which this "snap- 
through" occurs can become smaller and smaller. In other words, if the system is subject to a disturbance with $h_{1}<0$, however small, it will snap-through to the lower branch $A_{0}^{(2)}$ before $\mu$ has reached the bifurcation value 0 .

The restriction $h_{1}<0$ is in practice not significant. In a laboratory situation both positive and negative disturbances can be expected to occur, and it requires only one negative disturbance to cause snap-through. In an experiment it might be possible to control the magnitude, $H$, say, of all possible disturbances, but it seems highly improbable that one could control their sign. Our discussion suggests, however, that no matter how small $H$ is, snap-through from the null solution to the lower branch will eventually take place.

We can summarize these results in the following way.

Let $H>0$ be arbitrarily small. Then there exists $\bar{\mu}=\bar{\mu}(H) \in(\hat{\mu}, 0)$ such that, for each $\mu \in(\bar{\mu}, 0)$, there is an $h(x)$ with $\|h(x)\| \leqslant H$ for which the solution of the initialboundary-value problem

$$
\left.\begin{array}{rl}
\frac{\partial u}{\partial t} & =\frac{\partial^{2} u}{\partial x^{2}}+u+\varepsilon^{2}\left(\mu u-a u^{2}-u^{3}\right) \\
(0, t) & =u(\pi, t)=0, \quad u(x, 0)=h(x)
\end{array}\right\}
$$

tends as $t \rightarrow \infty$ to $u^{(2)}(x)$, where

$$
u^{(2)}(x)=A_{0}^{(2)} \sin x+O\left(\varepsilon^{2}\right)
$$

is a steady solution of (3.2).

Suppose now that, somewhere in the interval $\hat{\mu}<\mu<0$, the system occupies the solution $A_{0}^{(2)}$ as a result of snap-through. The next point to consider is whether and under what circumstances it will snap back to the null solution. The appropriate initial-boundary-value problem for disturbances in this case is obtained by setting $u(x, t)=u^{(2)}(x)+U(x, t)$ in (3.2). We obtain

$$
\left.\begin{array}{rl}
\frac{\partial U}{\partial t} & =\frac{\hat{o}^{2} U}{\partial x^{2}}+U+\varepsilon^{2}\left(\mu U-2 a u^{(2)} U-a U^{2}-3 u^{(2) 2} U-3 u^{(2)} U^{2}-U^{3}\right), \\
(0, t) & =U(\pi, t)=0, \quad U(x, 0)=h(x) .
\end{array}\right\}
$$

This system can be analysed in precisely the same way as was done in Section 2, and it is unnecessary to present the details. In fact the results for (3.4) can be deduced from a suitable interpretation of the results of Section 2 . If $h_{1}<0$ the solution of (3.4) tends to the null solution (that is, the lower branch) as $t \rightarrow \infty$. Similar behaviour occurs if $0<h_{1}<A_{0}^{(1)}-A_{0}^{(2)}$. It follows that all disturbances to the lower solution are damped if their norm is less than $A_{0}^{(1)}-A_{0}^{(2)}$, and this quantity increases monotonically as $\mu$ increases from $\hat{\mu}$ towards 0 . Therefore if we 
set a limit $H$ to the magnitude of disturbances there will be a range of values of $\mu$ close to 0 for which the system cannot snap back to the (original) null solution. In other words, we have the following result:

Let $H>0$ be as in the previous result. Then there exists $\overline{\bar{\mu}}=\overline{\bar{\mu}}(H) \in(\hat{\mu}, 0)$ such that for each $\mu \in(\overline{\bar{\mu}}, 0)$ the solution of the initial-boundary-value problem (3.4) tends to 0 as $t \rightarrow \infty$ for all $h(x)$ such that $\|h(x)\| \leqslant H$.

We conclude therefore that under normal circumstances the system will snapthrough to and remain on the lower branch, which is in this sense "more stable" than the upper branch. Asymmetry, even though small, can play a decisive role in determining the selection of alternative linearly stable branches.

\section{Acknowledgements}

This work was performed while the author was a participant in the Northwestern University Summer Workshop in Applied Mathematics during July-August 1978. The author is indebted to the Department of Engineering Sciences and Applied Mathematics, Northwestern University, for its hospitality and support. The work was supported in part by the Army Research Office, Durham, under grant DAAG 29-77-G-0222.

\section{References}

[1] T. B. Benjamin, "Bifurcation phenomena in steady flows of a viscous fluid", Proc. Roy. Soc. A 359 (1978), 1-43.

[2] S. H. Davis and L. A. Segel, "Effects of surface curvature and property variations on cellular convection", Phys. Fluids 11 (1968), 470-476.

[3] B. J. Matkowsky, "A simple nonlinear dynamic stability problem", Bull. Amer. Math. Soc. $76(1970), 620-625$.

[4] L. A. Segel and J. T. Stuart, "On the question of the preferred mode in cellular thermal convection", J. Fluid Mech. 13 (1962), 289-306.

[5] H. F. Weinberger and S. Rosenblat, "The continuous behaviour of bifurcation in the presence of finite noise", (1980) (to appear).

Department of Mathematics

University of Melbourne

Parkville

Victoria 3052 